\title{
Electromechanical Activation Time in the Prediction of Discharge Outcomes in Patients Hospitalized with Acute Heart Failure Syndrome
}

\author{
Tze-Fan Chao ${ }^{1,3}$, Shih-Hsien Sung ${ }^{1,3}$, Hao-Min Cheng ${ }^{2,3}$, Wen-Chung Yu ${ }^{1,3}$, \\ Kang-Ling Wang ${ }^{1,3}$, Chi-Ming Huang ${ }^{1,3}$ and Chen-Huan Chen $^{2-4}$
}

\begin{abstract}
Objective Left ventricular systolic time intervals, including pre-ejection period (PEP), ejection time (ET), and their ratio (PEP/ET), is determined by systolic and diastolic function and ventriculo-arterial coupling. We investigated the usefulness of the electromechanical activation time [(EMAT) PEP minus isovolumic contraction time] in the prediction of cardiac mortality or re-hospitalization for heart failure in patients with the acute heart failure syndrome (AHFS).

Patients and Methods A total of 45 patients ( $71.9 \pm 16.1$ years old) hospitalized for AHFS were enrolled. Systolic time intervals and EMAT normalized by cardiac cycle length (\%) were measured separately by separate automated acoustic devices. All parameters were assessed within 24 hours of admission, before discharge, and 2 weeks after discharge.

Results During a mean follow-up of $242 \pm 156$ days, 20 patients (44\%) incurred adverse cardiovascular events including 18 re-hospitalizations for heart failure and 2 cardiovascular deaths. Using uni-variate Cox regression analysis, at admission \%EMAT and \%PEP, pre-discharge \%EMAT, and post-discharge \%EMAT significantly predicted post-discharge cardiovascular events with hazard ratios and $95 \%$ confidence intervals of $1.75(1.13-2.70), 1.67(1.02-2.70), 1.87(1.10-3.17)$ and 2.50 (1.58-3.97) per 1-SD increment, respectively. The predictions remained significant after adjustment for age, sex, left ventricular ejection fraction, E/E’ by Doppler echocardiography, and serum $\mathrm{N}$-terminal pro-brain natriuretic peptide.

Conclusion EMAT measured during the hospitalization course is useful in the prediction of cardiovascular outcomes in patients with AHFS independent of left ventricular ejection fraction, $\mathrm{E}^{\prime} \mathrm{E}^{\prime}$, and serum N-terminal pro-brain natriuretic peptide.
\end{abstract}

Key words: systolic time intervals, electromechanical activation time, acute decompensated heart failure, prognosis

(Inter Med 49: 2031-2037, 2010)

(DOI: 10.2169/internalmedicine.49.3944)

\section{Introduction}

Acute heart failure syndrome (AHFS) indicates gradual or rapid change in heart failure signs and symptoms resulting in a need for urgent therapy (1). The majority of patients appear to respond well to initial medical therapies (1). How- ever, post-discharge mortality and re-hospitalization rates are high and may reach $10 \%$ to $20 \%$ and $20 \%$ to $30 \%$, respectively, within 3 to 6 months (2). Thus, prognostic stratification of AHFS patients before or soon after discharge is desirable to optimize individual treatment and to reduce the overall health care burden related to heart failure.

In patients hospitalized due to AHFS, low systolic blood

\footnotetext{
${ }^{1}$ Department of Medicine, Taipei Veterans General Hospital, Taipei, Taiwan, ${ }^{2}$ Department of Medical Research and Education, Taipei Veterans General Hospital, Taipei, Taiwan, ${ }^{3}$ Cardiovascular Research Center, National Yang-Ming University, Taipei, Taiwan and ${ }^{4}$ Department of Public Health, National Yang-Ming University, Taipei, Taiwan

Received for publication May 13, 2010; Accepted for publication June 11, 2010

Correspondence to Dr. Chen-Huan Chen, chench@vghtpe.gov.tw
} 
pressure on admission, renal insufficiency, hyponatremia, and use of positive inotropic therapy are associated with inhospital and short term outcomes (3-5). In both chronic heart failure and patients hospitalized due to AHFS, plasma $\mathrm{N}$-terminal pro-brain natriuretic peptide (NT-proBNP) is a powerful marker of short-term and long-term mortality and heart failure hospitalization, independent of conventional predictors (6).

Left ventricular (LV) systolic time intervals, including pre-ejection period (PEP), ejection time (ET), and their ratio (PEP/ET), is determined by the LV systolic and diastolic function and ventriculo-arterial coupling $(7,8)$. The electromechanical activation time (EMAT, the interval from beginning of electrical activation of LV to the onset of the first heart sound, encompassing both electromechanical delay and the portion of ventricular contraction from onset to mitral valve closure) constitutes the initial portion of PEP (9), and is useful in the detection of LV systolic dysfunction and resolving ambiguous values of B-type natriuretic peptide levels in patients with suspected heart failure $(10,11)$. However, the role of EMAT in the risk stratification of AHFS remains to be determined. Therefore, the goal of the present study was to evaluate the usefulness of EMAT in the prediction of post-discharge outcomes independent of common clinical predictors for patients hospitalized due to AHFS.

\section{Methods}

\section{Participants and study design}

AHFS was defined as either new-onset heart failure or decompensation of chronic, established heart failure with symptoms sufficient to warrant hospitalization. Hospitalized AHFS patients were assessed by an experienced cardiologist for ascertainment of the diagnosis and enrollment of the present study. Patients with atrial fibrillation, significant aortic valvular disease, or electrocardiographic left or right bundle branch block were not enrolled. The study protocol adhered to the principles of the Declaration of Helsinki and was approved by the Institutional Review Board at Taipei Veterans General Hospital. Informed consent was obtained from all patients.

Systolic time intervals and EMAT were performed within 24 hours after admission, before discharge, and two weeks after discharge. A comprehensive echocardiographic examination and measurement of serum NT-proBNP were also performed before discharge. NT-proBNP was measured by an enzyme-linked immunosorbent assay micro-titer plate (Roche Diagnostics, Basel, Switzerland) (7, 12).

Systolic time intervals were measured by an automated device (VP-2000, Colin Corporation, Komaki, Japan). Details of the measurement had been described in our previous works $(7,8)$. Briefly, the device performed electrocardiography, phonocardiography, and pulse volume recording on four extremities (ankles and arms). Pulse volume recording at cuff pressure of $60 \mathrm{mmHg}$ was recorded for 10 seconds.
The beat-by beat interval of the initial deflection of electrocardiographic Q wave to the first high frequency vibration of the aortic component of the second heart sound interval (QS2) and the interval of the beginning upstroke to the dicrotic notch of the pulse volume trace of the right arm (ET) were determined. PEP was calculated by subtracting ET from QS2 (8). ET and PEP were the averages of around 10 beats.

After the measurement of systolic time intervals, a 10second audio-electrocardiographic tracing was obtained and algorithmically analyzed using the Audicor device (Inovise Medical, Inc., Portland, OR, USA) (11). EMAT was determined by measuring the interval from the initial deflection of the electrocardiographic Q wave to the peak component of the first heart sound complex.

To correct for heart rate, PEP, ET, and EMAT were divided by the R-R interval to determine the percent of time in the cardiac cycle occupied by each interval and yield \% PEP, \%ET, and \%EMAT, respectively (11). All measurements of the systolic time intervals and EMAT were obtained automatically from the two devices and the operators who performed the examinations were blinded to the other clinical data.

Doppler and M-mode echocardiographic examination was performed using a multifrequency transducer incorporated in a SONOS 5500 Echocardiograph (Hewlett Packard, Inc., Agilent Technologies, Andover, MA, USA) according to the American Society of Echocardiography criteria. LV ejection fraction (EF) was calculated using the M-mode measurements. Stroke volume (SV) was measured by pulse wave Doppler echocardiography. From the mitral inflow velocity tracings, peak velocity (E) and deceleration time (EDT) of the early inflow wave, and peak velocity (A) of late inflow wave at atrial contraction were measured. E/E' was calculated as the ratio of $\mathrm{E}$ and early peak ( $\left.\mathrm{E}^{\prime}\right)$ of mitral annulus velocity obtained from the apical 4-chamber view in the septal and lateral mitral valve annulus. The acquired values were averaged (13). The measurements were performed by an experienced investigator who was blinded to the other clinical data.

Ventriculo-arterial coupling was assessed using the framework of the ratio of effective arterial elastance (Ea) to endsystolic elastance (Ees) (8). Ees was estimated with a previously proposed single-beat method employing brachial systolic blood pressure (SBP) and diastolic blood pressure (DBP), SV, EF, and an estimated normalized ventricular elastance at arterial end-diastole $[\mathrm{E}(\mathrm{Nd})]$ : Ees $=[\mathrm{DBP}-(\mathrm{E}$ (Nd) $\left.\left.{ }^{*} \mathrm{SBP}{ }^{*} 0.9\right)\right] /\left[\mathrm{E}(\mathrm{Nd}){ }^{*} \mathrm{SV}\right]$, where $\mathrm{E}(\mathrm{Nd})$ was estimated from a group-averaged value adjusted for individual contractile/loading effects (14). Ea was the ratio of LV end-systolic blood pressure (Pes), which is approximated to value of $\mathrm{SBP} \times 2 / 3+\mathrm{DBP} \times 1 / 3$, to the value of SV (15).

After admission, all patients were stabilized by appropriate medical therapy and were discharged. All patients met the following discharge criteria: optimal volume status achieved, transition from intravenous to oral diuretic com- 
pleted, free from oxygen support, and patient education completed. They were followed up 2 weeks after discharge and then monthly at outpatient clinics or by telephone consultations to check if there were any adverse cardiovascular events including re-hospitalization for heart failure, non-fatal myocardial infarction, non-fatal stroke, and cardiovascular death. These adverse cardiovascular events were confirmed by review of medical records. The period of event-free survival was defined as the interval between the pre-discharge hemodynamic survey and the date of adverse events or the end of follow-up.

\section{Statistical analysis}

Data are presented as mean values and standard deviations for normally distributed continuous variables, medians and interquartile ranges for skewed data, and proportions for categorical variables. Differences between continuous values were assessed using an unpaired 2-tailed t test for normally distributed continuous variables, the Mann-Whitney ranksum test for skewed variables, and chi-square testing for nominal variables. The event-free survival curves were plotted via Kaplan-Meier method with statistical significance examined by the log-rank test. Cox regression analysis was used to identify the predictors for future cardiovascular events. All statistical significances were set at $\mathrm{p}<0.05$ and all statistical analyses were carried out by SPSS ver17.0 analytical software (SPSS Inc., Chicago, Il, USA).

\section{Results}

We enrolled 45 patients ( $89 \%$ male; mean age 71.9 years; range 24 to $92 ; 37.8 \%$ with $\mathrm{LV} \mathrm{EF} \geq 50 \%$; $60 \%$ de novo heart failure hospitalization). During a follow-up of $242 \pm 156$ days, 20 patients incurred adverse cardiovascular events, including 18 re-hospitalizations for heart failure and 2 cardiovascular deaths. Their baseline characteristics and predischarge echocardiographic parameters were similar except that the patients with post-discharge cardiovascular events had significantly greater $\mathrm{E}^{-\mathrm{E}^{\prime}}$ and higher levels of NTproBNP as compared with those without events (Table 1).

Serial measurements of systolic time intervals and \% EMAT at admission, before discharge, and 2 weeks after discharge are presented in Table 2. \%PEP was significantly longer in patients with than without events at admission but not before or after discharge. In contrast, \%EMAT was significantly longer in patients with than without events at admission, before and after discharge.

By Cox regression analysis, pre-discharge log-transformed value of NT-ProBNP levels significantly predicted postdischarge cardiovascular events with hazard ratios and $95 \%$ confidence intervals of 2.19 (1.24-3.84) per 1-SD increment. Similarly, at-admission \%PEP, at-admission \%EMAT, predischarge \% EMAT, and post-discharge \% EMAT significantly predicted post-discharge cardiovascular events with hazard ratios and 95\% confidence intervals of 1.67 (1.02$2.70), 1.75$ (1.13-2.70), 1.87 (1.10-3.17), and 2.50 (1.58-
3.97) per 1-SD increment, respectively. After adjustment for age, Sex, LV EF, E/E' and NT-proBNP, at-admission, predischarge and post-discharge \%EMAT remained significant predictors for post-discharge cardiovascular events (Table 3). In contrast, NT-proBNP level was not a significant predictor in the multivariate analysis after adjustment for age, sex, LV $\mathrm{EF}, \mathrm{E} / \mathrm{E}^{\prime}$ and at admission, pre-discharge and post-discharge $\%$ EMAT with hazard ratios and $95 \%$ confidence intervals of 1.86 (0.88-3.92), 1.97 (0.89-4.36), and 2.15 (0.97-4.76) per 1-SD increment, respectively. At a cut-point of 15 (16), Kaplan-Myer survival analysis showed that both high predischarge \%EMAT (Fig. 1A) and high post-discharge \% EMAT (Fig. 1B) significantly predicted cardiovascular events. The positive and negative predictive values of predischarge \%EMAT at a cut-point of 15 for cardiovascular events were $66.67 \%$ and $63.64 \%$, respectively. The positive and negative predictive values of post-discharge \%EMAT at a cut-point of 15 for cardiovascular events were $75 \%$ and $72.4 \%$, respectively. The specificities of the pre-discharge and post-discharge \%EMAT were both $84 \%$.

Correlation coefficients of \%EMAT, \%PEP, \%ET, and $\mathrm{PEP} / \mathrm{ET}$ with LV and arterial function parameters and logtransformed NT-proBNP levels are shown in Table 4. All 4 time-interval parameters were significantly correlated with LV EF. In addition, \%EMAT significantly was correlated with $\mathrm{Ea}$ and $\mathrm{Ea} / \mathrm{Ees}$, whereas PEP/ET was significantly correlated with Ea/Ees. Furthermore, only \%EMAT was significantly correlated with NT-proBNP levels.

\section{Discussion}

To our knowledge, this study was the first attempt to investigate the usefulness of time-interval related LV functional parameters, including the conventional systolic time intervals and EMAT in the prediction of discharge outcomes in patients hospitalized with AHFS. Our preliminary but encouraging results showed that none of the conventional systolic time intervals parameters independently predicted outcomes in AHFS patients. In contrast, values of at-admission, pre-discharge and post-discharge \%EMAT were independently predictive of cardiovascular events in AHFS patients after discharge.

\section{Systolic time intervals}

Systolic time intervals are useful in the quantitative assessment of myocardial performance in patients with severe heart failure and ischemic heart disease (17), and after cardiac resynchronization therapy (18). It is well-established that PEP is prolonged and ET is shortened in patients with congestive heart failure, and PEP/ET correlates with LV EF (19). We further extended that PEP is prolonged and $\mathrm{PEP} / \mathrm{ET}$ is increased in patients with diastolic heart failure (8). In addition, PEP/ET is useful in the identification of heart failure patients with abnormal ventriculo-arterial coupling (8). Despite the good correlation between systolic time intervals and LV dysfunction, there is considerable overlap 
Table 2. Systolic Time Intervals and Electromechanical Activation Time Related Variables at Admission, Predischarge, and 2-Week after Discharge, and the Univariate Hazard Ratios and 95\% Confidence Intervals Per One-standard Deviation for Event Prediction

\begin{tabular}{|c|c|c|c|c|}
\hline Variables & $\begin{array}{l}\text { With events } \\
(\mathrm{n}=20)\end{array}$ & $\begin{array}{l}\text { No events } \\
(\mathrm{n}=25)\end{array}$ & $\begin{array}{c}\text { Hazard ratio } \\
(95 \% \mathrm{CI})\end{array}$ & $\mathrm{p}$ values \\
\hline \multicolumn{5}{|l|}{ At admission } \\
\hline$\%$ EMAT & $15.8 \pm 4.1^{* *}$ & $12.6 \pm 3.1$ & $1.75(1.13-2.70)$ & 0.012 \\
\hline$\%$ РEP & $15.7 \pm 4.0^{*}$ & $13.4 \pm 4.2$ & $1.67(1.02-2.70)$ & 0.04 \\
\hline$\%$ ET & $33.7 \pm 3.5$ & $32.8 \pm 5.2$ & $0.62(0.72-1.72)$ & 0.623 \\
\hline PEP/ET & $0.48 \pm 0.14$ & $0.42 \pm 0.15$ & $1.44(0.87-2.34)$ & 0.157 \\
\hline \multicolumn{5}{|l|}{ Predischarge } \\
\hline$\%$ EMAT & $14.1 \pm 4.1^{*}$ & $12.8 \pm 2.8$ & $1.87(1.10-3.17)$ & 0.021 \\
\hline$\%$ PEP & $13.5 \pm 3.6$ & $13.2 \pm 3.8$ & $1.05(0.70-1.60)$ & 0.819 \\
\hline$\% \mathrm{ET}$ & $30.6 \pm 5.0$ & $32.6 \pm 4.0$ & $0.88(0.59-1.31)$ & 0.517 \\
\hline $\mathrm{PEP} / \mathrm{ET}$ & $0.45 \pm 0.16$ & $0.41 \pm 0.13$ & $1.13(0.74-1.74)$ & 0.570 \\
\hline \multicolumn{5}{|c|}{ After discharge } \\
\hline$\%$ EMAT & $15.4 \pm 4.3 * *$ & $12.6 \pm 2.6$ & $2.50(1.58-3.97)$ & $<0.001$ \\
\hline$\%$ PEP & $14.3 \pm 4.0$ & $13.3 \pm 3.4$ & $1.21(0.78-1.87)$ & 0.392 \\
\hline$\%$ ET & $31.3 \pm 4.9$ & $33.5 \pm 7.1$ & 0.87 (0.50-1.49) & 0.867 \\
\hline PEP/ET & $0.46 \pm 0.15$ & $0.41 \pm 0.12$ & $1.16(0.76-1.79)$ & 0.497 \\
\hline \multicolumn{5}{|c|}{$\begin{array}{l}*: \mathrm{p}<0.05 \text { as compared with patients without event. } \\
* *: \mathrm{p}<0.01 \text { as compared with patients without event. } \\
\% \mathrm{EMAT}=\text { normalized electromechanical activation time; } \% \mathrm{PEP}=\text { normalized } \\
\text { pre-ejection period; } \% \mathrm{ET}=\text { normalized ejection time; } \mathrm{PEP} / \mathrm{ET}=\text { ratio of } \mathrm{PEP} \text { over } \\
\mathrm{ET} .\end{array}$} \\
\hline
\end{tabular}

Table 3. Multi-variate Hazard Ratios and 95\% Confidence Intervals Per One-standard Deviation for Systolic Time Intervals and EMAT in the Prediction of Cardiovascular Events

\begin{tabular}{|c|c|c|}
\hline Variables & Hazard ratio $(95 \% \mathrm{CI})$ & $\mathrm{p}$ value \\
\hline \multicolumn{3}{|l|}{ At admission } \\
\hline \%EMAT & $2.27(1.21-4.28)$ & 0.011 \\
\hline \%РEP & $2.07(0.98-4.43)$ & 1.10 \\
\hline \multicolumn{3}{|c|}{ Before discharge } \\
\hline \%EMAT & $1.91(1.06-3.64)$ & 0.041 \\
\hline \multicolumn{3}{|c|}{ After discharge } \\
\hline$\%$ EMAT & $2.91(1.70-5.01)$ & $<0.001$ \\
\hline $\begin{array}{l}\text { Variables we } \\
\% \text { EMAT }=\mathrm{n} \\
\text { pre-ejection } \mathrm{p} \\
\text { ET. }\end{array}$ & $\begin{array}{l}\mathrm{x}, \mathrm{LV} \mathrm{EF}, \mathrm{E} / \mathrm{E}^{\prime} \text { and } \mathrm{NT}- \\
\text { lanical activation time; } \\
\text { ized ejection time; } \mathrm{PEP}\end{array}$ & $\begin{array}{l}\text { lalized } \\
\text { PEP over }\end{array}$ \\
\hline
\end{tabular}

nificantly predicted events, the significance disappeared after multivariate adjustment.

\section{EMAT}

EMAT (Q-S1 interval) and the subsequent isovolumic contraction time constitute the PEP. PEP measures the amount of time that LV requires to generate sufficient force to first close the mitral valve (EMAT) and then open the aortic valve (isovolumic contraction time) (22). In patients with myocardial infarction and heart failure with impaired myocardial function, both EMAT and isovolumic contraction time are prolonged $(9,20)$. However, PEP is a preloaddependent parameter (23). In subjects with normal heart function, reduction in preload and LV volume results in prolongation of PEP, which is mainly due to the prolonged isovolumic contraction time resulting from decreased LV filling pressure and increased pressure gradient across the aortic valve (24). Conversely, an increase in preload shortens PEP because of the decreased trans-aortic pressure gradient and the increased contractility of the heart according to Starling's law. In contrast, patients with heart failure having raised LV filling pressures show a decrease in PEP and PEP/ ET in response to preload reduction (23). On the other hand, recent studies demonstrated that \%EMAT was not associated with LV end diastolic pressure $(16,25)$, indicating less preload sensitivity with \%EMAT.

An abnormal \%EMAT $(\geq 15)$ had $44 \%$ sensitivity, 94\% specificity, and a 7.0 likelihood ratio for the identification of patients with LV dysfunction (LV end-diastolic pressure $>15$ $\mathrm{mmHg}$ and $\mathrm{LV} \mathrm{EF}<50 \%$ ) (16). In addition, in patients with a grey-zone brain natriuretic peptide levels (100-500 pg/ $\mathrm{mL}$ ), an abnormal \%EMAT substantially improved the prediction for LV dysfunction (16). In the present study, \% EMAT was significantly correlated with LV EF and Ea but not with $\mathrm{E} / \mathrm{E}^{\prime}$. Ea is considered a lumped index of arterial load, including arterial stiffness (26). In 72 stable heart failure patients with an averaged LV EF of 53\%, elevated arterial stiffness was a risk factor for re-hospitalization or car- 

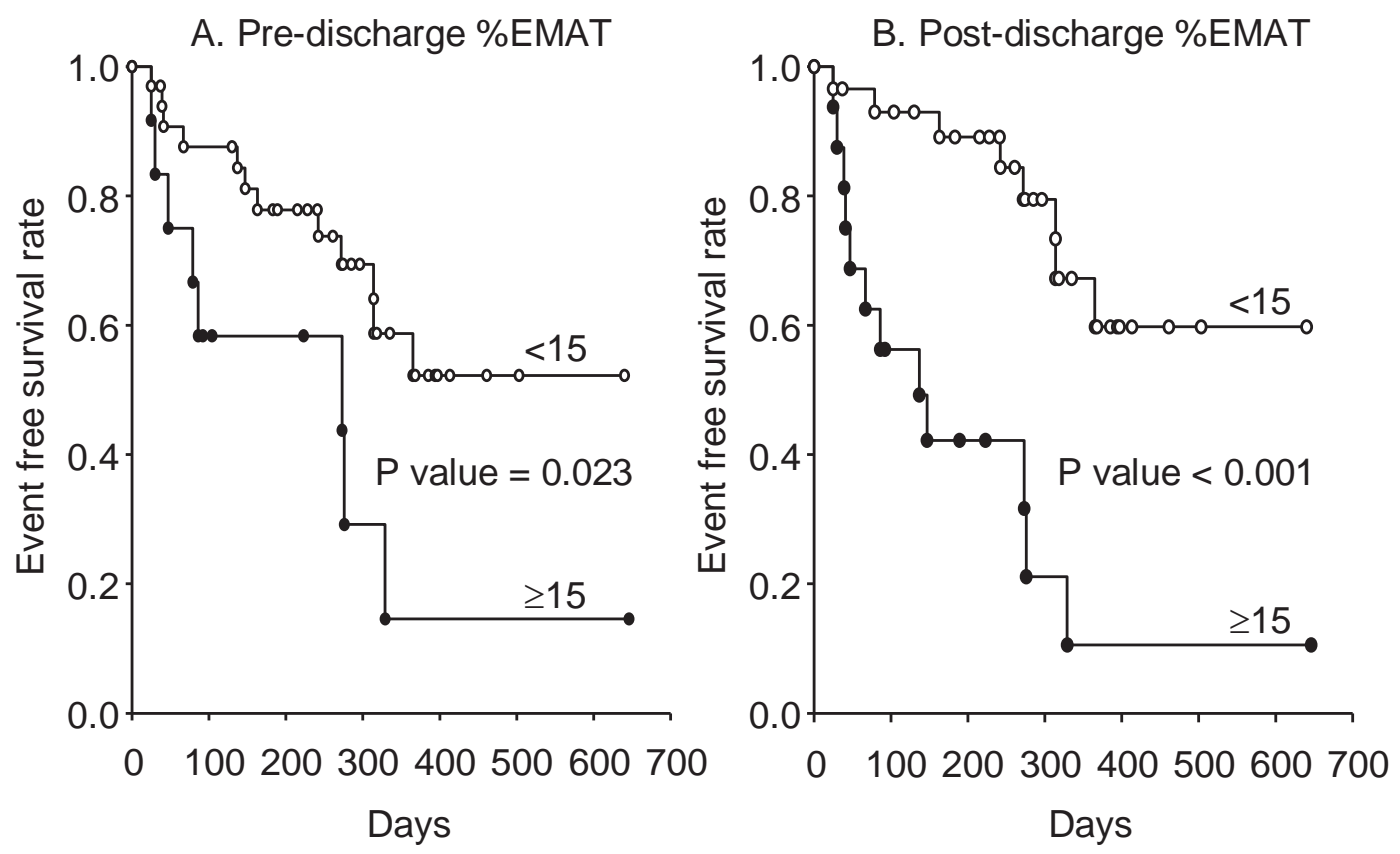

Figure 1. Survival curves for acute heart failure syndrome patients after discharge. KaplanMeier survival analysis showed a significantly reduced event-free survival for patients with prolonged pre-discharge (A) or post-discharge (B) \%EMAT (>15). EMAT: normalized electromechanical activation time.

Table 4. Correlation Coefficients of Systolic Time Intervals EMAT with Pre-discharge Parameters of Left Ventricular and Arterial Function and NT-proBNP

$\begin{array}{lccccc} & \mathrm{EF}, \% & \mathrm{E} / \mathrm{E}^{\prime} & \mathrm{Ea}, \mathrm{mmHg} / \mathrm{L} & \mathrm{Ea} / \mathrm{Ees} & \begin{array}{c}\text { NT-proBNP, } \\ \mathrm{pg} / \mathrm{mL}\end{array} \\ \text { \%EMAT } & -0.506^{* * *} & 0.124 & 0.331^{*} & 0.311^{*} & 0.291^{*} \\ \% \mathrm{PEP} & -0.432^{* * *} & -0.090 & 0.101 & 0.309 & 0.034 \\ \% \mathrm{ET} & 0.355^{*} & -0.016 & 0.018 & -0.296 & -0.062 \\ \mathrm{PEP} / \mathrm{ET} & -0.575^{* * *} & 0.032 & 0.168 & 0.410^{* *} & 0.115 \\ *: \mathrm{p}<0.05 ; * * \text { p }<0.01 ; * * *: \mathrm{p}<0.005 & \\ \mathrm{Ea}=\text { effective arterial elastance; Ea/Ees = ratio of Ea over Ees; Ees = left ventricular } \\ \text { end-systolic elastance; EF = ejection fraction; E/E' = the ratio of early peak (E) of } \\ \text { mitral inflow and early peak (E') of mitral annulus velocity; \%EMAT = normalized } \\ \text { electromechanical activation time; \%ET = normalized ejection time; NT-proBNP = } \\ \text { N-terminal pro-brain natriuretic peptide; PEP/ET = ratio of PEP over ET; \%PEP = } \\ \text { normalized pre-ejection period. }\end{array}$

diac death (27). E/E' correlated well with simultaneously estimated pulmonary capillary wedge pressure (13) and LV filling pressure. $28 \mathrm{E} / \mathrm{E}^{\prime}>15$ has been considered to be the best Doppler predictor of increased mean LV diastolic pressure (28). Furthermore, \%EMAT but not systolic time intervals was significantly correlated with NT-proBNP levels (Table 4). Therefore, our results may support that \%EMAT is sensitive to both LV systolic function and arterial stiffness while insensitive to change of preload, and may be a better predictor of outcome for the discharged AHFS patients than systolic time intervals.

\section{Limitations of the present study}

Although the results of the present study were statistically significant and clinically relevant, confirmation in future studies with a sufficiently large number of patients with clearly defined systolic and diastolic heart failure is required.

\section{Conclusions}

Quick measurement of \%EMAT by acoustic cardiography during the hospitalization or early after discharge may be useful in the prediction of future cardiovascular events in patients hospitalized for AHFS.

\section{Acknowledgement}

This work was supported by the intramural grants (V97C1101, V98C1-028, and V99C1-091) from Taipei Veterans General Hospital, Taiwan, Republic of China. 


\section{References}

1. Gheorghiade M, Zannad F, Sopko G, et al. Acute heart failure syndromes: current state and framework for future research. Circulation 112: 3958-3968, 2005.

2. Yancy CW, Lopatin M, Stevenson LW, De Marco T, Fonarow GC. Clinical presentation, management, and in-hospital outcomes of patients admitted with acute decompensated heart failure with preserved systolic function: a report from the Acute Decompensated Heart Failure National Registry (ADHERE) Database. J Am Coll Cardiol 47: 76-84, 2006.

3. Gheorghiade M, Abraham WT, Albert NM, et al. Systolic blood pressure at admission, clinical characteristics, and outcomes in patients hospitalized with acute heart failure. JAMA 296: 2217-2226, 2006.

4. Gheorghiade M, Abraham WT, Albert NM, et al. Relationship between admission serum sodium concentration and clinical outcomes in patients hospitalized for heart failure: an analysis from the OPTIMIZE-HF registry. Eur Heart J 28: 980-988, 2007.

5. Abraham WT, Adams KF, Fonarow GC, et al. In-hospital mortality in patients with acute decompensated heart failure requiring intravenous vasoactive medications: an analysis from the Acute Decompensated Heart Failure National Registry (ADHERE). J Am Coll Cardiol 46: 57-64, 2005.

6. Januzzi JL, van Kimmenade R, Lainchbury J, et al. NT-proBNP testing for diagnosis and short-term prognosis in acute destabilized heart failure: an international pooled analysis of 1256 patients: the International Collaborative of NT-proBNP Study. Eur Heart J 27: 330-337, 2006.

7. Cheng HM, Chuang SY, Hsu PF, Chou P, Chen CH. Systolic time intervals revisited: correlations with $\mathrm{N}$-terminal pro-brain natriuretic peptide in a community population. Heart Vessels 20: 256263, 2005.

8. Cheng HM, Yu WC, Sung SH, Wang KL, Chuang SY, Chen CH. Usefulness of systolic time intervals in the identification of abnormal ventriculo-arterial coupling in stable heart failure patients. Eur J Heart Fail 10: 1200, 2008.

9. Diamant B, Killip T. Indirect assessment of left ventricular performance in acute myocardial infarction. Circulation 42: 579-592, 1970.

10. Roos M, Toggweiler S, Jamshidi P, et al. Noninvasive detection of left ventricular systolic dysfunction by acoustic cardiography in cardiac failure patients. J Card Fail 14: 310-319, 2008.

11. Zuber M, Kipfer P, Attenhofer Jost CH. Usefulness of acoustic cardiography to resolve ambiguous values of B-type natriuretic Peptide levels in patients with suspected heart failure. Am J Cardiol 100: 866-869, 2007.

12. Hobbs FD, Davis RC, Roalfe AK, Hare R, Davies MK, Kenkre JE. Reliability of N-terminal pro-brain natriuretic peptide assay in diagnosis of heart failure: cohort study in representative and high risk community populations. BMJ 324: 1498-1502, 2002.
13. Cheng HJ, Zhang ZS, Onishi K, Ukai T, Sane DC, Cheng CP. Upregulation of functional beta(3)-adrenergic receptor in the failing canine myocardium. Circ Res 89: 599-606, 2001.

14. Chen $\mathrm{CH}$, Fetics B, Nevo E, et al. Noninvasive single-beat determination of left ventricular end-systolic elastance in humans. J Am Coll Cardiol 38: 2028-2034, 2001.

15. Kelly RP, Ting CT, Yang TM, et al. Effective arterial elastance as index of arterial vascular load in humans. Circulation 86: 513-521, 1992.

16. Moyers B, Shapiro M, Marcus GM, et al. Performance of phonoelectrocardiographic left ventricular systolic time intervals and Btype natriuretic peptide levels in the diagnosis of left ventricular dysfunction. Ann Noninvasive Electrocardiol 12: 89-97, 2007.

17. Weissler AM. Current concept in cardiology: systolic time intervals. N Engl J Med 296: 321-324, 1977.

18. Baker C, Love CJ, Moeschberger ML, Orsinelli DA, Yamokoski L, Leier CV. Time intervals of cardiac resynchronization therapy in heart failure. Am J Cardiol 94: 1192-1196, 2004.

19. Garrard CL Jr, Weissler AM, Dodge HT. The relationship of alterations in systolic time intervals to ejection fraction in patients with cardiac disease. Circulation 42: 455-462, 1970.

20. Weissler AM, Harris WS, Schoenfeld CD. Systolic time intervals in heart failure in man. Circulation 37: 149-159, 1968.

21. Hodges M, Halpern BL, Friesinger GC, Dagenais GR. Left ventricular preejection period and ejection time in patients with acute myocardial infarction. Circulation 45: 933-942, 1972.

22. Erne P. Beyond auscultation-acoustic cardiography in the diagnosis and assessment of cardiac disease. Swiss Med Wkly 138: 439-452, 2008.

23. Khanna PK, Shah PM, Kramer DH, Schaefer RA, Tager I. Effects of altered preload on left ventricular systolic time intervals in acute myocardial infarction. Br Heart J 35: 1102-1108, 1973.

24. Weissler AM, Harris WS, Schoenfeld CD. Bedside technics for the evaluation of ventricular function in man. Am J Cardiol 23: 577$583,1969$.

25. Efstratiadis S, Michaels AD. Computerized acoustic cardiographic electromechanical activation time correlates with invasive and echocardiographic parameters of left ventricular contractility. J Card Fail 14: 577-582, 2008.

26. Borlaug BA, Melenovsky V, Redfield MM, et al. Impact of arterial load and loading sequence on left ventricular tissue velocities in humans. J Am Coll Cardiol 50: 1570-1577, 2007.

27. Meguro T, Nagatomo Y, Nagae A, et al. Elevated arterial stiffness evaluated by brachial-ankle pulse wave velocity is deleterious for the prognosis of patients with heart failure. Circ J 73: 673-680, 2009.

28. Ommen SR, Nishimura RA, Appleton CP, et al. Clinical utility of Doppler echocardiography and tissue Doppler imaging in the estimation of left ventricular filling pressures: A comparative simultaneous Doppler-catheterization study. Circulation 102: 1788-1794, 2000.

(C) 2010 The Japanese Society of Internal Medicine http://www.naika.or.jp/imindex.html 